Check for updates

Cite this: RSC Adv., 2019, 9, 3208

\title{
Investigation on the chirality mechanism of chiral carbon quantum dots derived from tryptophan $\uparrow$
}

\author{
Yingying Wei, ${ }^{\text {ab }}$ Lin Chen, ${ }^{\text {bc }}$ Junli Wang, ${ }^{\text {bc }}$ Xuguang Liu, (D)*ab Yongzhen Yang (D) *bc \\ and Shiping $Y u^{\text {bd }}$
}

Chiral carbon quantum dots (CQDs) with chirality, fluorescence and biocompatibility were synthesized by a one-step method with L-/D-tryptophan (L-/D-Trp), as both carbon source and chiral source. Levogyration-/dextrorotation-CQDs (L-/D-CQDS) were characterized by transmission electron microscopy, Fourier transform infrared spectrometry, ultraviolet-visible absorption, excitation and emission spectrometry and circular dichroism (CD) spectrometry. Results show that L-CQDs and DCQDs present similar spherical morphology, functional groups and optical properties. The CD signal, around 220, 240 and $290 \mathrm{~nm}$ are opposite and symmetric, which conclusively demonstrates that LCQDs and D-CQDs are enantiomers. Besides the CD signal around $220 \mathrm{~nm}$ from the inheritance of L-/DTrp, two new chiral signals around 240 and $290 \mathrm{~nm}$ were induced by chiral environment.

Received 23rd November 2018 Accepted 12th January 2019

DOI: $10.1039 / \mathrm{c} 8 \mathrm{ra09649j}$

rsc.li/rsc-advances by researchers in the field of biology in the past few years. However, their application in biological environment was limited because of the lack of chiral ligands for preparing chiral QDs, and high cost and toxicity. Carbon quantum dots (CQDs), a new class of carbon nanoparticles with size less than $10 \mathrm{~nm}, 4,5$ have fluorescence properties similar to QDs, but do not contain toxic metal elements, and have outstanding advantages such as easy functionalization, low cost, very low toxicity and favorable biocompatibility. ${ }^{5-8}$

Recently, research on chiral CQDs has attracted great attention and achieved some good achievements. The researches on chiral CQDs indicated that levogyration-CQDs (LCQDs) and dextrorotation-CQDs (D-CQDs) present different biological effects. For instance, L-CQDs show chiralitydependent enhancement in cellular glycolysis, whereas $\mathrm{D}$ CQDs have no similar effect, suggesting that chiral CQDs are selective in energy metabolism of cells; ${ }^{3}$ D-CQDs are able to enhance photosynthesis better and accumulate more carbohydrate in mung bean plants, which can be potentially used as fertilizer for agricultural applications; ${ }^{9}$ L-CQDs dramatically remodel amyloid beta-42 (A $\beta 42)$ secondary structure and fibril morphologies, as well as inhibit $\mathrm{A} \beta 42$ cytotoxicity and membrane interactions. ${ }^{10}$ In a word, the biological effect is closely related to chiral characteristics of materials, and chiral CQDs especially have promising application in biology.

Up to now, the synthesis of chiral CQDs can be divided into two categories, including one-step and two-step approach. In 2016, Suzuki et al. firstly reported the generation of chirality on graphene quantum dots (GQDs). ${ }^{11}$ GQDs were synthesized and $\mathrm{L}-/ \mathrm{D}-$ cysteine $(\mathrm{L}-\mathrm{D}-\mathrm{c}$ - 5 ) moieties were subsequently covalent attached to their edges. In two-step approach, CQDs were prepared firstly, and then modified by chiral ligand to obtain Taiyuan 030001, China

$\dagger$ Electronic supplementary information (ESI) available. See DOI: 10.1039/c8ra09649j 


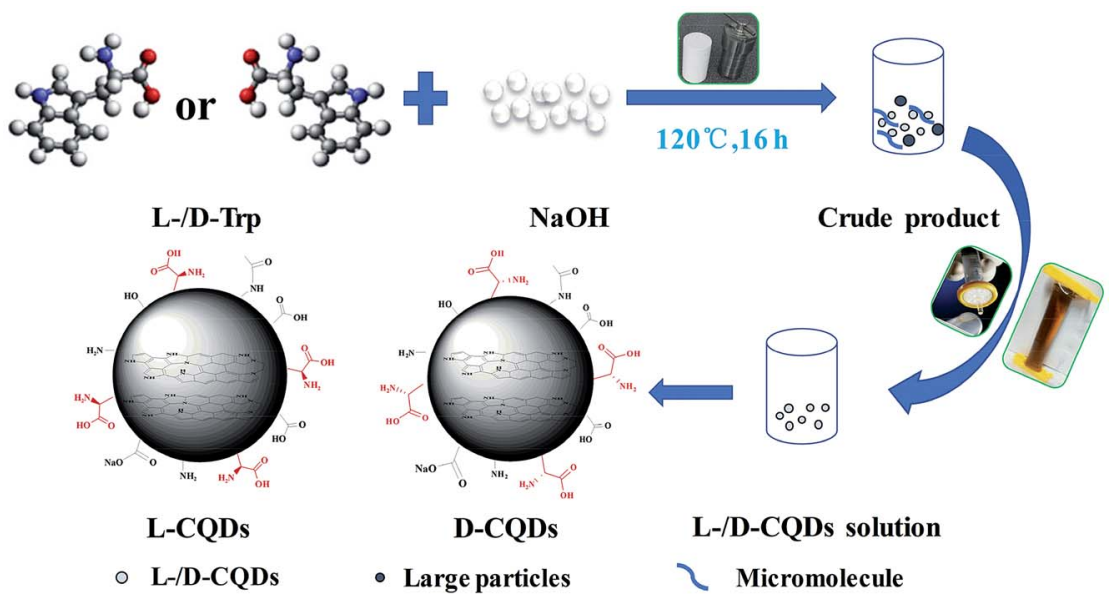

Fig. 1 Schematic for the synthesis of L-/D-CQDs by one-step hydrothermal method.

chiral CQDs. This process is relatively complicated and the conditions are severe. Nevertheless, in one-step method, chiral CQDs were obtained using carbon source and chiral ligand in a system, and it is a new challenge to synthesize chiral CQDs with simultaneous functionalization, doping or capping with other chiral molecules. Through unremitting efforts, researchers have developed a one-step method to directly prepare chiral CQDs. By taking citric acid as carbon source and $\mathrm{L}-/ \mathrm{D}$-cys as chiral source, ${ }^{9,12}$ or $\mathrm{L}-/ \mathrm{D}-\mathrm{cys},{ }^{13} \mathrm{~L}-/ \mathrm{D}-\mathrm{lysine}{ }^{10}$ and other chiral amino acids as raw materials to synthesize chiral CQDs, chiral inheritance or chiral induction from amino acids was realized onto CQDs. ${ }^{14}$ However, the chirality origin of these chiral CQDs still remains unclear.

In view of this, in order to clarify the chirality mechanism of chiral CQDs prepared by amino acids, chiral CQDs were synthesized by a one-step hydrothermal method using L-/Dtryptophan (L-/D-Trp), a widely available and low-cost natural resource, as both carbon source and chiral source, and the chirality origin in CQD structure was revealed.

\section{Experimental}

\subsection{Raw materials}

L-Trp and levogyration-alanine (L-Ala) were provided by Tianjin Guangfu Fine Chemical Research Institute. D-Trp, dextrorotation-alanine (D-Ala) and indole were obtained from Shanghai McLean Biochemical Technology Co. Ltd. $\mathrm{NaOH}$ flakes were purchased from Tianjin Mainland Chemical Reagent Factory, which were used without any further purification. Cellulose dialysis bags with 1000 Da molecular mass were purchased from Shanghai Yuanye Biotechnology Co. Ltd.

\subsection{Synthesis of $\mathrm{L}-/ \mathrm{D}-\mathrm{CQDS}$}

At first, $0.5 \mathrm{~g}$ of $\mathrm{L}-/ \mathrm{D}-\mathrm{Trp}$ and $0.09 \mathrm{~g}$ of $\mathrm{NaOH}$ were sufficiently dissolved in $15 \mathrm{~mL}$ of water under ultrasonication for $5 \mathrm{~min}$. Then, the above-mentioned solution was transferred to a $100 \mathrm{~mL}$ Teflon-sealed autoclave and heated at $120^{\circ} \mathrm{C}$ for $16 \mathrm{~h}$. The resultant solution, which was brown and transparent, was filtered to remove larger carbon nanoparticles by syringe filter (pore size: $0.22 \mu \mathrm{m}$ ). After that, purification was conducted through a dialysis bag (molecular weight cut off $1000 \mathrm{Da}$ ) for four days to remove unreacted raw materials as well as low molecular weight by-products. The dialysis fluid was dried in freeze dryer to obtain the products. Fig. 1 shows schematic for the synthesis of $\mathrm{L}-/ \mathrm{D}-\mathrm{CQDS}$ by one-step hydrothermal method.

\subsection{Chirality origin analysis}

$\mathrm{L}-\mathrm{D}$-Trp can be decomposed into indole and L-/D-Ala under hydrothermal, high pressure and alkaline condition, L-/D-Ala and indole were involved in the reaction. L-/D-Ala creates a chiral environment to induce achiral chromophores distortion. Therefore, in order to further study the chiral origin of $\mathrm{L}^{-} / \mathrm{D}-$ CQDs, three parallel experiments were designed. Meanwhile, to unify the quality of the raw materials among the contrast experiments, the three groups of raw materials for contrast experiments were: first group ( $0.28 \mathrm{~g}$ of indole), second group $(0.22 \mathrm{~g}$ of $\mathrm{L}-/ \mathrm{D}-\mathrm{Ala})$ and third group $(0.28 \mathrm{~g}$ of indole $+0.22 \mathrm{~g}$ of $\mathrm{L}-/$ D-Ala). The reaction procedures were the same as Section 2.2.

\subsection{Characterization}

Transmission electron microscopy (TEM) and high-resolution TEM images (HRTEM) were taken on a JEOL JEM-2100 microscope with an accelerating voltage of $60 \mathrm{kV}$. Fourier transform infrared (FT-IR) spectra were recorded in the form of $\mathrm{KBr}$ pellets with a BRUKER TENSOR 27 spectrometer. X-ray photoelectron spectroscopy (XPS) measurements were conducted on a Kratos AXIS ULTRA DLD X-ray photoelectron spectrometer with mono $\mathrm{X}$-ray source $\mathrm{Al} \mathrm{K} \alpha$ excitation $(1486.6 \mathrm{eV})$. The excitation and emission spectra of $\mathrm{L}-/ \mathrm{D}-\mathrm{CQDS}$ aqueous solution were collected on a Horiba Fluoromax-4 luminescence spectrometer using Xe lamp as excitation source. The circular dichroism spectra (CD) and ultraviolet-visible (UV-vis) absorption spectra of samples aqueous solution were obtained on a JASCO J-1500 spectropolarimeter. 


\section{Results and discussion}

\subsection{Morphology and structure of the $\mathrm{L}-/ \mathrm{D}-\mathrm{CQDS}$}

By comparing the characterization results of L-CQDs and DCQDs, it is found that their morphology and structure are similar. Therefore the text mainly includes the information for L-CQDs, while the TEM and XPS spectra about D-CQDs are shown in the ESI. $\dagger$ As shown in Fig. 2(a), as-prepared L-CQDs are well dispersed spherical nanoparticles morphology with average size of $4.0 \mathrm{~nm}$ in diameter. The HRTEM image shows L-CQDS possess lattice spacing of $0.26 \mathrm{~nm}$, which corresponds to the (100) facets of graphite (Fig. 2(b)). ${ }^{15}$ As for D-CQDs, as shown in Fig. $\mathrm{S} 1, \uparrow$ the size distribution range is similar to that of $\mathrm{L}_{\text {-CQDS. }}$ The average particle size is about $3.0 \mathrm{~nm}$ slightly smaller than that of L-CQDs. As shown in Fig. S1(b), $\dagger$ the D-CQDs particles also have a lattice fringe of $0.26 \mathrm{~nm}$.

The chemical structure of the obtained L-CQDs or D-CQDs was probed using XPS. The full-scan XPS spectrum of ${ }_{L}-C Q D s$ is depicted in Fig. 3(a), where O1s, C1s, N1s and Na1s peaks demonstrate that L-CQDs are principally composed of carbon, oxygen, nitrogen and sodium, which is consistent with the raw material. $\mathrm{NaOH}$ is involved in the reaction and may form sodium carboxylate. ${ }^{16}$ For L-CQDs, the expanded image of the C1s peak confirms the presence of $\mathrm{C}-\mathrm{C} / \mathrm{C}=\mathrm{C}(284.43 \mathrm{eV}), \mathrm{C}-\mathrm{N}$ $\left(\mathrm{sp}^{3}, 285.43 \mathrm{eV}\right), \mathrm{C}-\mathrm{O}\left(\mathrm{sp}^{3}, 286.65 \mathrm{eV}\right)$, and $\mathrm{C}=\mathrm{O}\left(\mathrm{sp}^{2}, 288.27 \mathrm{eV}\right)$ bonds (Fig. 3(b)). ${ }^{17-19}$ The N1s spectra present three peaks at 398.62, 399.17 and $400.57 \mathrm{eV}$, which are related to graphitic $\mathrm{N}$, pyridinic $\mathrm{N}$ and pyrrolic N, respectively (Fig. 3(c)). ${ }^{\mathbf{1 8 2 0 - 2 2}}$ The O1s spectra exhibit three peaks at 531.06, 531.84 and $532.73 \mathrm{eV}$, which are partly assigned to $\mathrm{O}-\mathrm{C}, \mathrm{O}=\mathrm{C}$, and $\mathrm{HO}-\mathrm{C} / \mathrm{C}-\mathrm{O}-\mathrm{C}$ groups, respectively (Fig. 3(d)). ${ }^{\mathbf{2 3} 24}$ These results demonstrate that as-prepared L-CQDs contain lots of functional groups on their surface. The chemical compositions of D-CQDs are similar to those of $\mathrm{L}$-CQDs, as shown in Fig. S2. $\dagger$

To further analyze the surface functional groups of L-CQDs and D-CQDs, their FT-IR spectra were compared with the raw material L-/D-Trp, as shown in Fig. 4. The broad band at $3402 \mathrm{~cm}^{-1}$ is attributed to $-\mathrm{OH} /-\mathrm{NH} /-\mathrm{NH}_{2}$ vibration of carboxylic acid and amine groups. ${ }^{25-27}$ Compared with that for $\mathrm{L}-/ \mathrm{D}-\mathrm{Trp}$, this band becomes wider, which may be induced by the association among hydrogen bonds. A small hump at $1662 \mathrm{~cm}^{-1}$ is assigned to $\mathrm{C}=\mathrm{O}$ stretching vibrations. ${ }^{6,27}$ The bands at $1600 \mathrm{~cm}^{-1}$ and $1400 \mathrm{~cm}^{-1}$ correspond to the asymmetric and symmetric stretching vibrations of COO- respectively. ${ }^{6,28}$ The bands at $1244 \mathrm{~cm}^{-1}$ and $1110 \mathrm{~cm}^{-1}$ represent the stretching of $-\mathrm{C}=\mathrm{ONH}$ and $\mathrm{C}-\mathrm{O}$, respectively. ${ }^{26,27}$ The presence of $-\mathrm{CONH}$ indicates the dehydration polymerization during the hydrothermal process. These results are in good accordance with XPS data. From the above data, there clearly exist that the oxygen- and nitrogen-containing functional groups on the surface of $\mathrm{L}_{-}$CQDs and D-CQDs.

\subsection{Optical properties of $\mathrm{L}-/ \mathrm{D}-\mathrm{CQDS}$}

Like most of CQDs, L-/D-CQDs also exhibit fluorescence properties. As shown in Fig. 5(a) and S3, there is no prominent difference in the UV-vis spectra of $\mathrm{L}_{\mathrm{L}} / \mathrm{D}$-CQDs and precursors. It can be clearly seen from the UV-vis spectra of $\mathrm{L}_{\text {-Trp }}$ and L-CQDS (Fig. 5) that L-CQDs have two obvious absorption peaks at 219 and $279 \mathrm{~nm}$, similar to raw material L-Trp. The strong absorption peak at $219 \mathrm{~nm}$ is attributed to $\pi-\pi^{*}$ transition of $\mathrm{C}=\mathrm{C},{ }^{29,30}$ while the weak absorption peak at $279 \mathrm{~nm}$ is ascribed to $n-\pi^{*}$ transition of $\mathrm{C}=\mathrm{O}^{, \mathbf{3 0 , 3 1}}$ and the latter part of absorption can generate fluorescence. ${ }^{5}$

The excitation and emission spectra of L-CQDs and D-CQDS are shown in Fig. 5(b) and S3(b), respectively. Compared with $\mathrm{L}^{-}$ Trp, L-CQDs have two excitation peaks, which are located at 287 and $363 \mathrm{~nm}$, and the maximum emission peak red-shifts from 355 to $476 \mathrm{~nm}$. Likewise, compared with D-Trp, D-CQDs have two excitation peaks, which are located at 290 and $363 \mathrm{~nm}$, and the maximum emission peak red-shifts from 361 to $476 \mathrm{~nm}$ (Fig. S3(b) $\dagger$ ).

To further study the optical properties of L- or D-CQDs, a series of emission spectra were obtained with different excitation wavelengths ranging from 320 to $400 \mathrm{~nm}$ with the increments of $20 \mathrm{~nm}$, as shown in Fig. 6 and S4. With the increase of excitation wavelength, the intensity of emission peak first increases and then decreases. And when the excitation wavelength is $360 \mathrm{~nm}$, the emission intensity reaches the maximum value, while the peak position of emission hardly
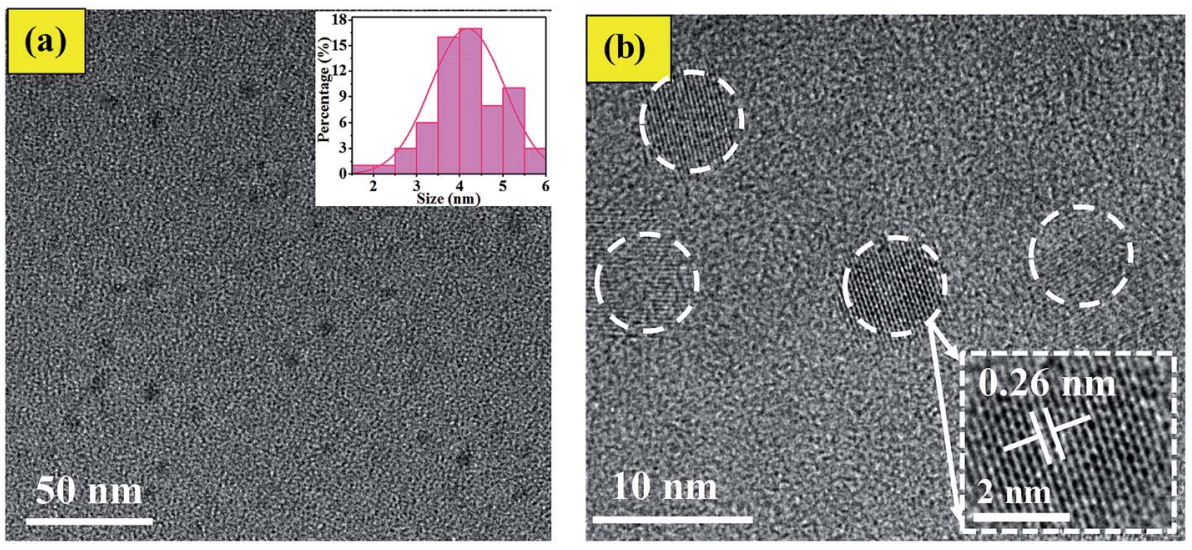

Fig. 2 (a) TEM image and particle size statistics, (b) HRTEM image of L-CQDs. 

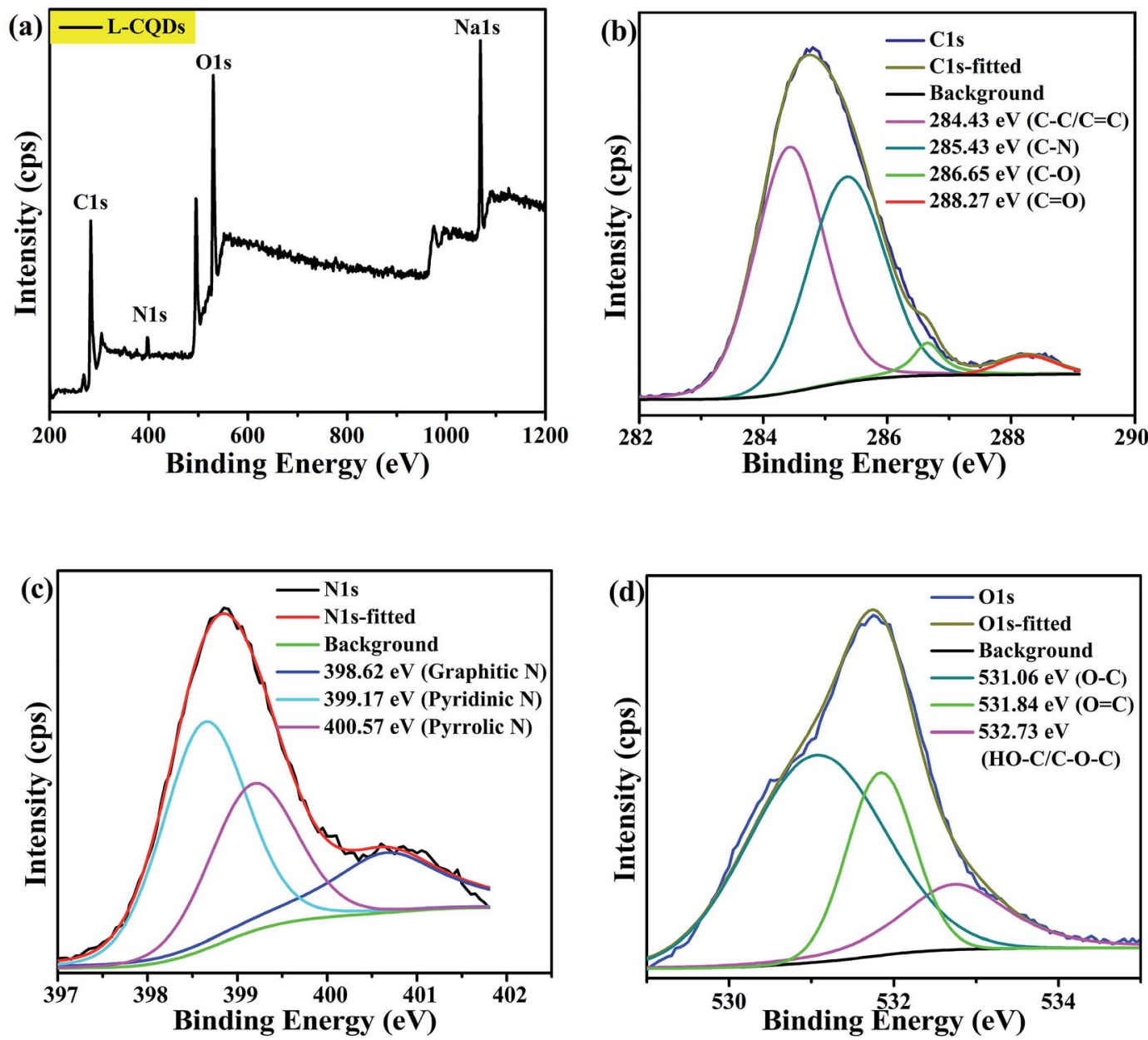

Fig. 3 (a) XPS, (b) C1s, (c) N1s and (d) O1s spectra of L-CQDs.

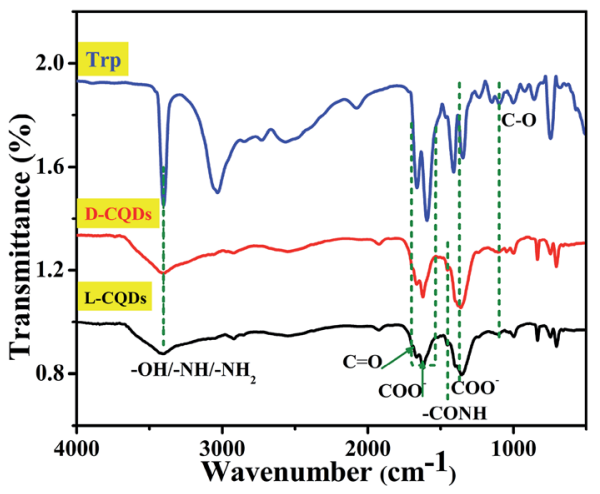

Fig. 4 FT-IR spectra of Trp, L-CQDs and D-CQDs.

changes, indicating L-CQDs exhibit excitation-independent nature, possibly resulting from a distribution of the same or similar emissive sites. ${ }^{18}$ The result of D-CQDs is consistent with that of L-CQDs, as shown in Fig. S4.†

The above analysis indicates that the product prepared by $\mathrm{L}-/$ D-Trp through hydrothermal reaction is zero-dimensional carbon nanoparticles with the size of less than $10 \mathrm{~nm}$ and has fluorescence characteristics, that is, CQDs. More importantly,

the chirality of the synthesized CQDs needs to be determined. Therefore, the chirality of the synthesized CQDs was analyzed by CD spectra. As shown in Fig. 7(a) and (b), CD spectra of $\mathrm{L}-/ \mathrm{D}-$ Trp and L-/D-CQDs show that the CD signals of L-Trp and D-Trp are symmetrical near $220 \mathrm{~nm}$, while the CD signals of L-CQDs and D-CQDs are symmetrical near 220, 240 and $290 \mathrm{~nm}$, and both CQDs and Trp exhibit enantiomeric isomerism, which proves that L-CQDs and D-CQDs are enantiomers. ${ }^{12}$ Obviously, the CD signal near $220 \mathrm{~nm}$ is generated by the intrinsic chiral chromophore, which inherits the chirality of L-/D-Trp..$^{12,13}$ The CD signals near 240 and $290 \mathrm{~nm}$ are generated by the influence of chiral environment, ${ }^{32}$ in which the CD signal near $240 \mathrm{~nm}$ is derived from the $\pi-\pi^{*}$ conjugation of the $\mathrm{sp}^{2}$-hybridized carbon network in $\mathrm{L}-/ \mathrm{D}-\mathrm{CQDS},{ }^{13,33}$ while the CD signal near $290 \mathrm{~nm}$ is attributed to $\pi-\pi^{*}$ transition between indole. ${ }^{34}$

\subsection{Synthetic mechanism of $\mathrm{L}-/ \mathrm{D}-\mathrm{CQDS}$}

Generally, the synthesis process of CQDs by hydrothermal method mainly includes dehydration, polymerization and carbonization. ${ }^{35}$ Therefore, the synthesis of $\mathrm{L}-\mathrm{D}-\mathrm{CQDS}$ goes through these processes. Indole and L-/D-Ala are produced because $\mathrm{L}-/ \mathrm{D}-\mathrm{Trp}$ is decomposed with water under hydrothermal, high pressure and alkaline condition. From above HRTEM, XPS 

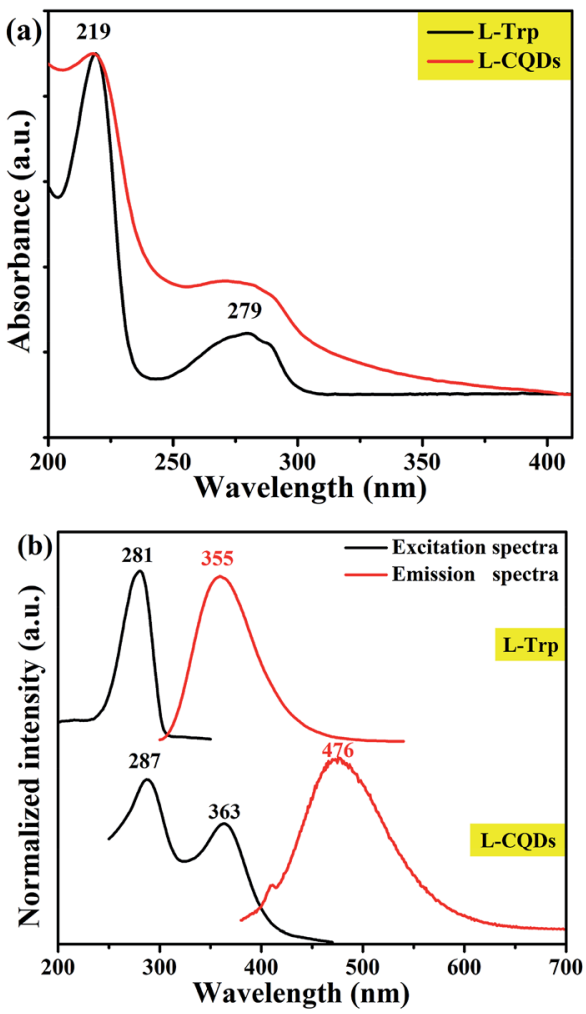

Fig. 5 UV-vis absorption (a), excitation and emission spectra (b) of LTrp and L-CQDs.

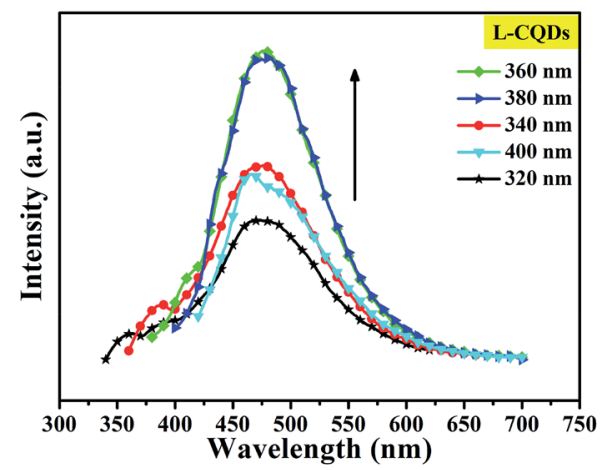

Fig. 6 Emission spectra of L-CQDs aqueous solutions at different excitation wavelengths.

and FT-IR characterizations, it can be seen that CQDs contain $\mathrm{sp}^{2}$-hybridized aromatic ring structure in their core and oxygenand nitrogen-containing functional groups on their surface. Therefore, it is inferred that the produced indoles are mainly involved in the formation of carbon core. $\mathrm{L}-/ \mathrm{D}-\mathrm{Ala}$ is undergone intramolecular or intermolecular dehydration and polymerization, and then carbonized to form CQDs. Fig. 8 depicts the

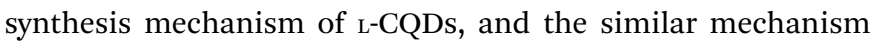
for D-CQDs is given in Fig. S5.† The synthesis mechanism is discussed in detail in following parts.

In this work, chiral CQDs were prepared by one-step hydrothermal method with chiral $\mathrm{L}^{-} / \mathrm{D}$-Trp and non-chiral reagent

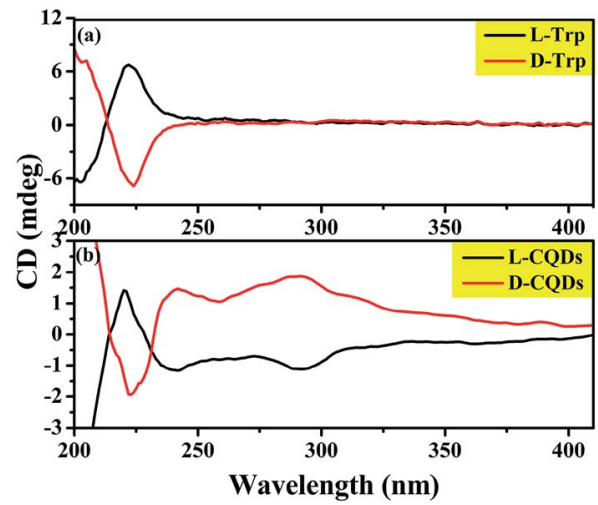

Fig. 7 CD spectra of L-/D-Trp (a) and L-/D-CQDs (b).

water as feedstock. Compared with current synthesis method of chiral CQDs, it is simple because no further surface functionalization is needed. Moreover, the chirality of CQDs is mainly generated from chiral L-/D-Trp, and retained at the quantum level. L-CQDs were synthesized from $\mathrm{L}^{-}$Trp and D-CQDs from DTrp. ${ }^{36}$ This process can be called asymmetric synthesis process, which is also known as chiral synthesis. According to the CD spectra of L-/D-CQDs, they present two additional CD signals near 240 and $290 \mathrm{~nm}$ compared with L-/D-Trp. The previous research of chiral CQDs has revealed that apart from preservation of chirality from Trp, chirality can also be induced by chiral environment for the distortion of achiral chromophores. ${ }^{32}$ Therefore, it is assumed that CD signals around 240 and $290 \mathrm{~nm}$ are induced by chiral environment.

In order to verify this assumption and the deposition of $\mathrm{L}^{-} / \mathrm{D}^{-}$ Trp under hydrothermal conditions, this work designed three parallel experiments were designed, as described in Section 2.3. UV-vis and CD spectra of the products were measured, as shown in Fig. 9. By comparison, it is found that the three products have a weak absorption peak at relatively long wavelengths. When indole is used as feedstock, a strong absorption peak appears at a relatively short wavelength, which is similar to the UV-vis spectra of $\mathrm{L}-/ \mathrm{D}-\mathrm{CQDS}$ (Fig. S3†), indicating that indole does participate in the reaction.

Fig. 9(d-f) are CD spectra of three groups of products. As confirmed by Fig. 9(d), when only indole takes part in reaction, the product has no chirality because the raw material itself has no chirality. However, as shown in Fig. 9(e), when only L-/D-Ala is reactant, a chiral environment is created on the product. A comparison between Fig. 9(e) and (f) indicates that, in addition to the chirality (around $205 \mathrm{~nm}$ ) of the product from L-/D-Ala, another chiral signal at $375 \mathrm{~nm}$ appears, obviously as a result of the distortion of achiral chromophores and the generation of chirality in a chiral environment. ${ }^{32}$ Because the products are prepared by L-/D-Ala and indole, which is different from the synthesis of $\mathrm{L}^{-} / \mathrm{D}-\mathrm{CQDS}$ by L-/D-Trp, a new chiral signal is deviated from 290 to $375 \mathrm{~nm}$ (Fig. 7). At the same time, the induction effect of chiral environment is further explained, that is in the synthesis process of L-/D-CQDs, CD signal near $290 \mathrm{~nm}$ is induced by chiral environment. ${ }^{34}$ 


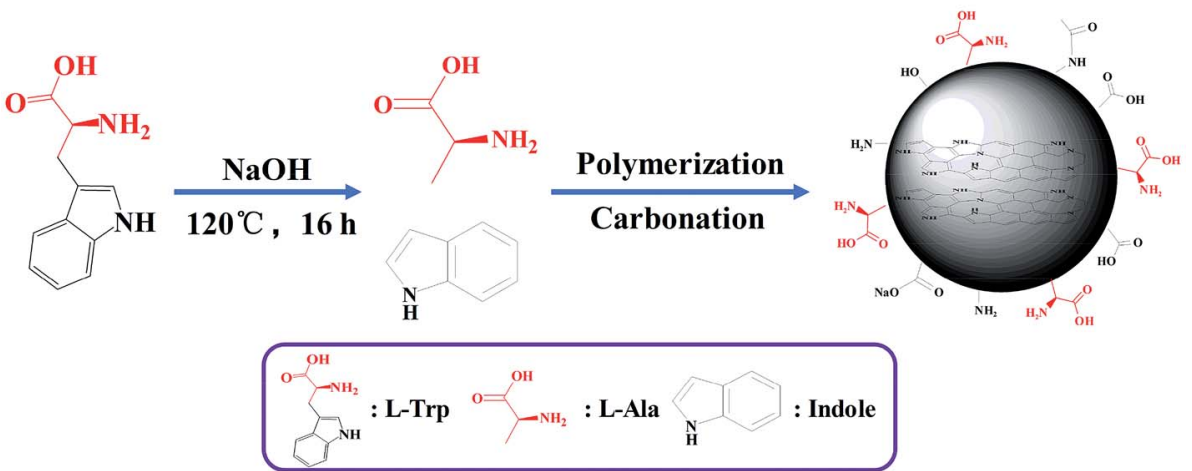

Fig. 8 Synthetic mechanism diagram of L-CQDs.
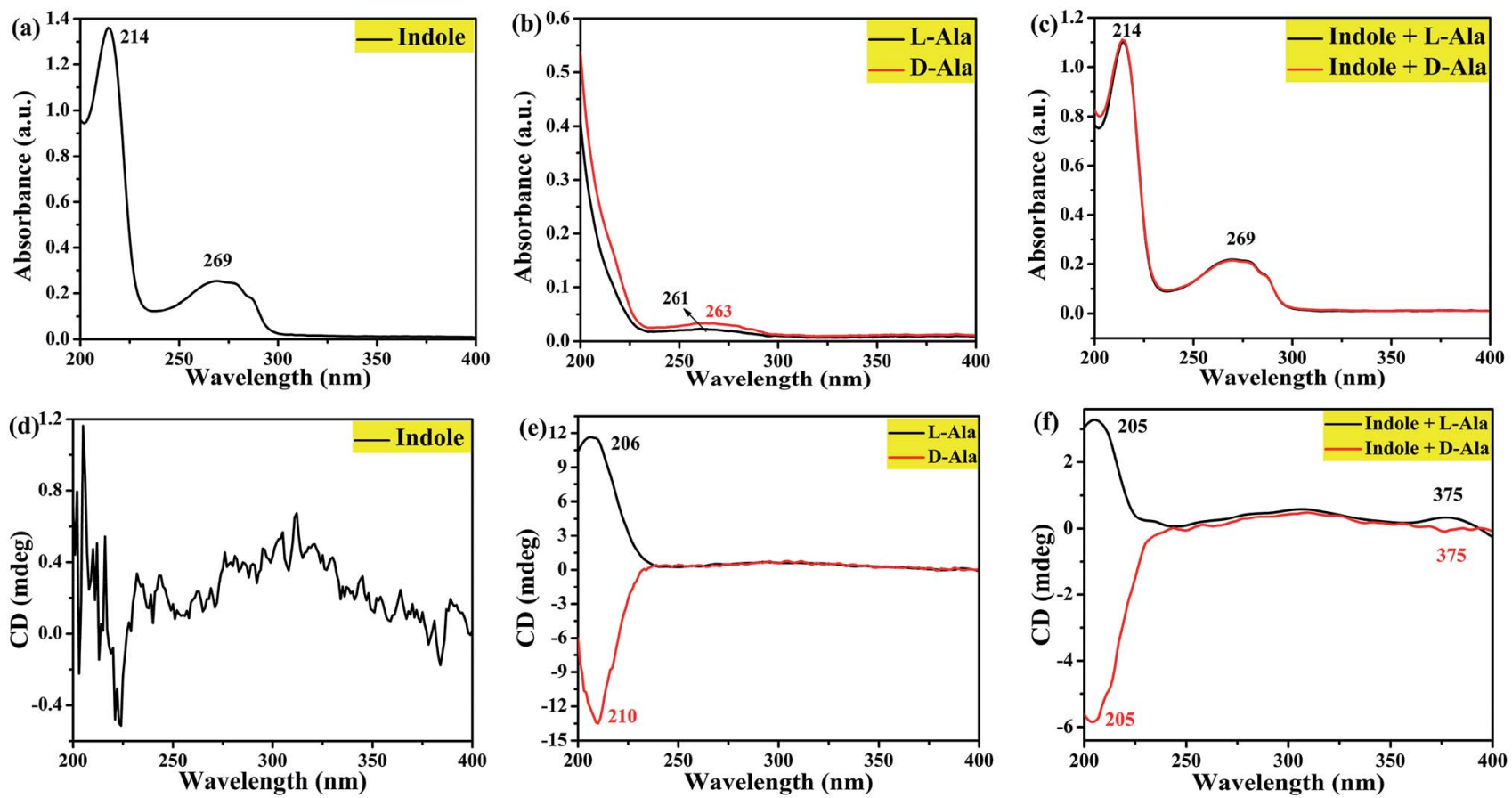

Fig. 9 UV-vis absorption ((a), (b) and (c)) and circular dichroism spectra ((d), (e) and (f)) of the hydrothermal products from reactants indole, L-/DAla, and indole + L-/D-Ala, respectively.

\section{Conclusion}

Chiral CQDs with fluorescence property were obtained by onestep hydrothermal method and their chirality mechanism was discussed. L-CQDs and D-CQDs exhibit similar morphology, structure and optical properties. Results show that the size distribution of $\mathrm{L}-/ \mathrm{D}-\mathrm{CQDS}$ around is $1.0-6.0 \mathrm{~nm}$. Their aqueous solution emits blue-green light under UV illumination at $365 \mathrm{~nm}$ and exhibits excitation-independent photoluminescence. The CD signals of L-CQDs and D-CQDs are symmetrical and opposite near 220, 240 and $290 \mathrm{~nm}$, which proves that L-CQDs and D-CQDs are enantiomers. Obviously, $\mathrm{L}^{-} /$ D-CQDs not only inherits the chirality of raw material L-/D-Trp on the surface of CQDs, but also adds two CD signals near 240 and $290 \mathrm{~nm}$. The CD signal near $240 \mathrm{~nm}$ is derived from the $\pi-\pi^{*}$ conjugation of the $\mathrm{sp}^{2}$-hybridized carbon network in $\mathrm{L}-/ \mathrm{D}-\mathrm{CQDS}$, while the CD signal near $290 \mathrm{~nm}$ is attributed to $\pi-\pi^{*}$ transition between indole. The chiral origin of chiral CQDs is understood, which not only guides the preparation of chiral CQDs, but also predicts the chiral site in CQDs. The resultant chiral CQDs may find application in chiral recognition, chiral detection and other fields.

\section{Conflicts of interest}

There are no conflicts to declare.

\section{Acknowledgements}

This work was supported by the National Key Research and Development Program of China (2017YFB0603104), the National Natural Science Foundation of China (51803148, 
U1710117, U1610255, U1607120), the Shanxi Provincial Key Research and Development Program (201703D321015-1), the Shanxi Provincial Key Innovative Research Team in Science and Technology (201605D131045-10) and the Shanxi Provincial Postgraduate Education Innovation Project (2018SY016).

\section{References}

1 F. P. Milton, J. Govan, M. V. Mukhina and Y. K. Gun'ko, Nanoscale Horiz., 2016, 1, 14-26.

2 M. P. Moloney, Y. K. Gun'ko and J. M. Kelly, Chem. Commun., 2007, 3900-3902.

3 F. Li, Y. Y. Li, X. Yang, X. X. Han, Y. Jiao, T. T. Wei, D. Y. Yang, H. P. Xu and G. J. Nie, Angew. Chem., 2018, 57, 2377-2382.

4 Y. B. Song, S. J. Zhu, S. T. Zhang, Y. Fu, L. Wang, X. H. Zhao and B. Yang, J. Mater. Chem. C, 2015, 3, 5976-5984.

5 R. Z. Zhang and W. Chen, Biosens. Bioelectron., 2014, 55, 8390.

6 Y. X. Hou, Q. J. Lu, J. H. Deng, H. T. Li and Y. Y. Zhang, Anal. Chim. Acta, 2015, 866, 69-74.

7 Q. Q. Fang, Y. Q. Dong, Y. M. Chen, C. H. Lu, Y. W. Chi, H. H. Yang and T. Yu, Carbon, 2017, 118, 319-326.

8 Z. Tian, X. T. Zhang, D. Li, D. Zhou, P. T. Jing, D. Z. Shen, S. N. Qu, R. Zboril and A. L. Rogach, Adv. Opt. Mater., 2017, 5, 1700416.

9 M. L. Zhang, L. L. Hu, H. B. Wang, Y. X. Song, Y. Liu, H. Li, M. W. Shao, H. Huang and Z. H. Kang, Nanoscale, 2018, 10, 12734-12742.

10 R. Malishev, E. Arad, S. K. Bhunia, S. Shaham-Niv, S. Kolusheva, E. Gazit and R. Jelinek, Chem. Commun., 2018, 54, 7762-7765.

11 N. Suzuki, Y. Wang, P. Elvati, Z. B. Qu, K. Kim, S. Jiang, E. Baumeister, J. Lee, B. Yeom, J. H. Bahng, J. Lee, A. Violi and N. A. Kotov, ACS Nano, 2016, 10, 1744-1755.

12 Y. L. Zhang, L. L. Hu, Y. Sun, C. Zhu, R. S. Li, N. Y. Liu, H. Huang, Y. Liu, C. Z. Huang and Z. H. Kang, RSC Adv., 2016, 6, 59956-59960.

13 L. L. Hu, Y. Sun, Y. J. Zhou, L. Bai, Y. L. Zhang, M. M. Han, H. Huang, Y. Liu and Z. H. Kang, Inorg. Chem. Front., 2017, 4, 946-953.

14 W. Ma, L. G. Xu, A. F. de Moura, X. L. Wu, H. Kuang, C. L. Xu and N. A. Kotov, Chem. Rev., 2017, 117, 8041-8093.

15 Y. F. Ding, J. X. Zheng, J. L. Wang, Y. Z. Yang and X. G. Liu, J. Mater. Chem. C, DOI: 10.1039/C8TC04887H.

16 D. Qu, M. Zheng, L. G. Zhang, H. F. Zhao, Z. G. Xie, X. B. Jing, R. E. Haddad, H. Y. Fan and Z. C. Sun, Sci. Rep., 2014, 4, 5294.
17 M. Xu, S. Xu, Z. Yang, M. Shu, G. He, D. Huang, L. Zhang, L. Li, D. Cui and Y. Zhang, Nanoscale, 2015, 7, 15915.

18 Y. Q. Dong, H. C. Pang, H. B. Yang, C. X. Guo, J. W. Shao, Y. W. Chi, C. M. Li and T. Yu, Angew. Chem., 2013, 52, 7800-7804.

19 J. L. Wang, F. Zhang, Y. L. Wang, Y. Z. Yang and X. G. Liu, Carbon, 2018, 126, 426-436.

20 S. Y. Lu, X. H. Zhao, S. J. Zhu, Y. B. Song and B. Yang, Nanoscale, 2014, 6, 13939-13944.

21 Q. Wang, C. L. Zhang, G. X. Shen, H. Y. Liu, H. L. Fu and D. X. Cui, J. Nanobiotechnol., 2014, 12, 58.

22 L. B. Tang, R. B. Ji, X. M. Li, G. X. Bai, C. P. Liu, J. H. Hao, J. Y. Lin, H. X. Jiang, K. S. Teng, Z. B. Yang and S. P. Lau, ACS Nano, 2014, 8, 6312-6320.

23 Y. Xu, M. Wu, Y. Liu, X. Z. Feng, X. B. Yin, X. W. He and Y. K. Zhang, Chemistry, 2013, 19, 2276-2283.

24 F. Zhang, X. T. Feng, Y. Zhang, L. P. Yan, Y. Z. Yang and X. G. Liu, Nanoscale, 2016, 8, 8618-8632.

25 N. Dhenadhayalan, K. C. Lin, R. Suresh and P. Ramamurthy, J. Phys. Chem. C, 2016, 120, 1252-1261.

26 X. Cui, L. Zhu, J. Wu, Y. Hou, P. Y. Wang, Z. N. Wang and M. Yang, Biosens. Bioelectron., 2015, 63, 506-512.

27 H. B. Xu, S. H. Zhou, L. L. Xiao, H. H. Wang, S. Z. Li and Q. H. Yuan, J. Mater. Chem. C, 2015, 3, 291-297.

28 W. B. Lu, X. Y. Qin, S. Liu, G. H. Chang, Y. W. Zhang, Y. L. Luo, A. M. Asiri, A. O. Al-Youbi and X. P. Sun, Anal. Chem., 2012, 84, 5351-5357.

29 M. Hassan, E. Haque, K. R. Reddy, A. I. Minett, J. Chen and V. G. Gomes, Nanoscale, 2014, 6, 11988-11994.

30 X. T. Zheng, A. Ananthanarayanan, K. Q. Luo and P. Chen, Small, 2015, 11, 1620-1636.

31 C. M. Luk, L. B. Tang, W. F. Zhang, S. F. Yu, K. S. Teng and S. P. Lau, J. Mater. Chem., 2012, 22, 22378.

32 D. Tedesco and C. Bertucci, J. Pharm. Biomed. Anal., 2015, 113, 34-42.

33 Q. Xin, Q. Liu, L. L. Geng, Q. J. Fang and J. R. Gong, Adv. Healthcare Mater., 2017, 6, 1601011.

34 S. M. Kelly and N. C. Price, Curr. Protein Pept. Sci., 2000, 1, 349-384.

35 J. Hou, W. Wang, T. Y. Zhou, B. Wang, H. Y. Li and L. Ding, Nanoscale, 2016, 8, 11185-11193.

36 M. J. Deka and D. Chowdhury, RSC Adv., 2017, 7, 5305753063. 